\title{
UNCERTAINTY CHARACTERISATION \& VALIDATION WITHIN ESA FIRE-CCI
}

\author{
Brennan J. ${ }^{1}$, Lewis, $P^{1}{ }^{1}$, Gomez-Dans, $J .^{1}$, Chernetskiy, $M .{ }^{1}$, \\ Chuvieco E. ${ }^{2}$, Lizundia J. ${ }^{2}$, Campagnolo M. ${ }^{3}$, Pereira J. ${ }^{3}$, Oom D. ${ }^{3}$ \\ ${ }^{1}$ UCL Dept. of Geography, University College London, London \\ \& National Centre For Earth Observation (NCEO) (UK) \\ ${ }^{2}$ Env. Rem. Sens. Group, University of Alcala, Alcalá, Spain \\ ${ }^{3}$ Dept. of Forestry, ISA, \\ Technical University of Lisbon, Portugal
}

\begin{abstract}
Uncertainty characterisation and validation are critical phases to generate any Essential Climate Variable (ECV), and therefore both have been included as key deliverables of the ESA CCI programme [1]. All products generated by the $\mathrm{CCI}$ are required to have an associated per pixel uncertainty characterisation. This paper describes both the uncertainty characterisation framework and the related uncertainty validation exercise of the Fire-CCI project.
\end{abstract}

Index Terms - Uncertainty, Burnt Area

\section{UNCERTAINTY DEFINITION WITHIN FIRE-CCI}

Burnt Area algorithms broadly consist of a classification into a binary Burnt/Unburnt product. As with all remote sensing retrievals these algorithms can be phrased within inverse theory:

$$
B=\mathbf{G}(d)
$$

Where the outputted burnt area product $B$ is determined by the data $d$ and the burnt area algorithm $\mathbf{G}$. Remote sensing measurements contain uncertainty [2]. As such the relationship above is better described by:

$$
B=\mathbf{G}(d)+v
$$

Where $v$ represents uncertainty in the value of the measurement $d$. Often the probability distribution function of $v$ is assumed normal such that the observation arrives as:

$$
d_{\text {observed }}=d_{\text {true }}+\mathcal{N}(0, \sigma)
$$

where $\sigma$ is an estimate of the observation uncertainty. Naturally the uncertainty in the observations leads to uncertainty in the retrieval of burnt area. To fully describe this uncertainty requires definitions of uncertainty at the relevant scales of the products. Within the ESA CCI programme their is an expectation of pixel-level uncertainty information [1]. Further, the
Fire-CCI products are also to be provided at a lower resolution Climate Model Grid (CMG) $\left(0.25^{\circ}\right)$.

\subsection{Pixel resolution}

At the pixel scale we define an appropriate uncertainty characterisation to be the probability that the pixel is burnt $P_{b}$. $P_{b}$ then provides an probabilistic confidence that the pixel is burnt given the observations. $P_{b}$ must follow the axiomatic laws of probability, e.g the probability that the pixel is not burnt $P_{u}$ is:

$$
P_{u}=1-P_{b}
$$

The full pixel level uncertainty is then described by a Bernoulli distribution $B$ :

$$
B=\left\{\begin{array}{lr}
P_{u}=1-P_{b}, & \text { if } \mathrm{B}=\text { Unburnt } \\
P_{b}, & \text { if } \mathrm{B}=\text { Burnt }
\end{array}\right\}
$$

\subsection{CMG resolution}

At the CMG scale the definition of uncertainty is different. Burnt area at the CMG is classically defined as the summation of the number of burnt pixels $N_{b}$. However to propagate the uncertainty to the $\mathrm{CMG}$ we suggest that the CMG burnt area $B_{\mathrm{CMG}}$ is better described by a Poisson binomial distribution. The Poisson binomial distribution describes the probability of $N$ independent Bernoulli distributions. As $N$ grows large the distribution is well described by a normal distribution with mean $\mu$ and standard deviation $\sigma$ :

$$
\mu=\sum_{i}^{N} p_{i} \quad \sigma=\sum_{i}^{N}\left(1-p_{i}\right) P_{i}
$$

This means that at the CMG scale each grid cell is approximated as a normal random variable which encodes the uncertainty in the burnt area. Crucially, all pixels are included in the calculation even if they have a low $P_{b}$. 


\section{UNCERTAINTY CHARACTERISATION EXERCISE}

An uncertainty validation methodology was developed for testing the uncertainty characterisation of algorithms within Fire-CCI. The purpose of the validation exercise was to assess the presently developed uncertainty characterisations within a framework which provides realistic estimates of the true product uncertainties. In this paper we address the validation of uncertainties for two algorithms within Fire-CCI.

\subsection{Algorithm uncertainty estimates $\hat{P}_{b}$}

The two algorithms make an estimate of the per-pixel uncertainty $\hat{P}_{b}$ in different manners. Algorithm A estimates $\hat{P}_{b}$ based primarily on a pixel's distance to the nearest active fire observation. While algorithm B defines $\hat{P}_{b}$ based on the distance to the classification boundary between the burnt and unburnt classes within the algorithm.

\subsection{Algorithm true uncertainty $P_{b}$}

Evidently the true uncertainty $P_{b}$ is determined by the confidence the algorithm places in the detection given the uncertainty in the data. To take account of the uncertainty in the observations, algorithms must marginalise over the probability distribution of the observation:

$$
P_{b}=\int_{d} P(d) \mathbf{G}(d) \delta d
$$

\subsection{Sampling Framework}

However the algorithms presently are unable to perform this integral. Instead a monte-carlo framework was developed to approximate this integral. We can derive an estimate of the true $P_{b}$ for an algorithm based on sampling from the data distribution $P(d)$.

Three test sites were selected, representing three significant pyromes for burnt area: savanna, boreal forest and tropical forest (which are shown in figure 1). An estimate of daily surface reflectance for each site was provided from the MODIS Collection 6 surface reflectance products (MOD/MYD09) .

To generate each realisation of the reflectance $R_{i}(\lambda)$, each recorded measurement from MODIS $R_{\text {MODIS }}(\lambda)$ was described by a multivariate normal distribution:

$$
R_{i}(\lambda)=R_{\text {MODIS }}(\lambda)+\mathcal{N}\left(\mathbf{0}, \mathbf{C}_{\lambda}\right)
$$

where the observation uncertainties are represented by the diagonal covariance matrix $\mathbf{C}_{\lambda}=\operatorname{diag}\left(\sigma_{i}^{2} \ldots \sigma_{\lambda}^{2}\right)$. Per channel uncertainties $\sigma_{\lambda}^{2}$ where provided from the MODIS uncertainty characterisation (see table 1) vermote2002atmospheric.

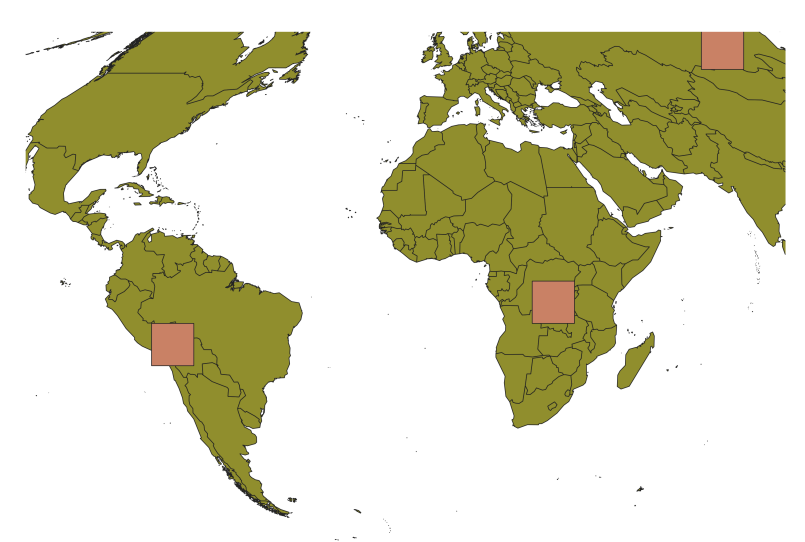

Fig. 1: Location of the selected test sites.

To consider that algorithms were properly representing the uncertainty in $P(d)$, three sets of realisations were computed for each site with three different levels of observational noise. To do this, the MODIS covariance matrix was scaled by a noise factor $c$, with the values of $0.5,1.0,1.5$. As $c$ increases, it would be expected that $P_{b}$ would tend more towards ignorance, as described by $P_{b}$ values approaching 0.5 . Both algorithms were run on the $N$ realisations of a remote sensing dataset sampled from $P(d)$ for each site.

As a result, for a pixel $p$ we have $N$ realisations of the product:

$$
P=[b, u, u, b, b, u, b, u, u, u, . .]
$$

where $b$ corresponds to a burnt detection and $u$ and unburnt detection. A good approximation to the true algorithm $P_{b}$ is then a function of the number of burnt draws to unburnt draws. When $B$ is defined as a Bernoulli variable, we can estimate the maximum likelihood estimate of $P_{b}$ from the function:

$$
\max \quad P_{b}^{B}\left(1-P_{b}\right)^{U}
$$

where $B$ and $U$ is the number of burnt and unburnt outputs respectively. $P_{b}$ is then simply:

$$
P_{b}=\frac{B}{N}
$$

Given the limited number of sample runs, this estimate may be poor [3]. Instead an adjusted estimate is provided by:

$$
P_{b}=\frac{\mathbf{B}(B+2, N-N+2)}{\mathbf{B}(N+1, n-B+1)}
$$

where $\mathbf{B}$ is the Beta function. Each realisation of $\hat{P}_{b}$ was then aggregated to the grid-scale along with the algorithm true $P_{b}$ as outlined in section 1.2. 
Table 1: Theoretical per-pixel uncertainty for MODIS channels. From: [?]

\begin{tabular}{rlllllll}
\hline MODIS channel & 1 & 2 & 3 & 4 & 5 & 6 & 7 \\
central $\lambda$ & $645 \mathrm{~nm}$ & $858 \mathrm{~nm}$ & $469 \mathrm{~nm}$ & $555 \mathrm{~nm}$ & $1240 \mathrm{~nm}$ & $1640 \mathrm{~nm}$ & $2130 \mathrm{~nm}$ \\
\hline$\sigma_{\lambda}^{2}$ & 0.004 & 0.015 & 0.003 & 0.004 & 0.013 & 0.010 & 0.006 \\
\hline
\end{tabular}

\section{RESULTS}

Figure 2 shows an example of the pixel level estimates of $P_{b}$ from two Fire-CCI algorithms compared to the true uncertainty characterisation. Primarily we see that the two algorithms show considerably different uncertainty estimates while having similar true uncertainties. Algorithm A shows high $\hat{P}_{b}$ for unburnt areas $(0.2-0.4)$ (with a low true $P_{b}$ ). Within the burn scars $\hat{P}_{b}$ is highest (typically 0.7-0.9) but still below the true uncertainty for these areas. Algorithm $\mathrm{B}$ matches more closely to the true uncertainty, especially in areas of low probability. However in the burn scars the estimated probabilities are considerably lower than the truth.
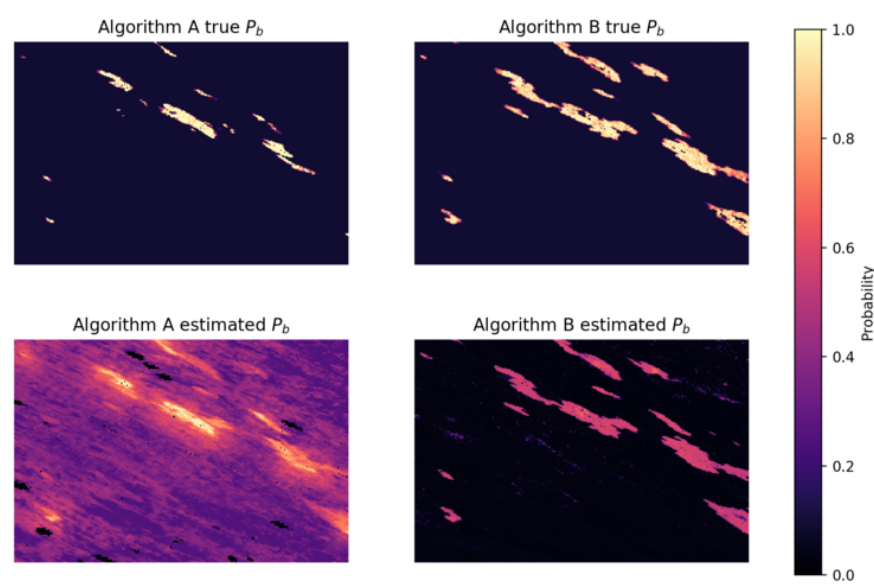

Fig. 2: Pixel level estimates of $P_{b}$ for two Fire-CCI algorithms over the boreal test site. Top) True $P_{b}$ derived from the sampling framework. Bottom) Algorithm estimate of $P_{b}$

Figure 3 shows the resulting CMG estimates of burnt area $\mathrm{km}^{2}$ from two Fire-CCI algorithms. The differences in the pixel level uncertainties clearly propagates to the CMG distributions. The over-estimation of uncertainties in algorithm A leads to a large over-estimation in the burnt area. Algorithm A also has an increased standard uncertainty relative to the true standard uncertainty given the data. Overall algorithm A estimate of burnt area does not match the true distribution well. Estimated distribution: $\mu: 23267 \mathrm{~km}^{2} \sigma: 13306 \mathrm{~km}^{2}$ vs the sampling-based distribution: $\mu: 6395 \mathrm{~km}^{2} \sigma: 4951 \mathrm{~km}^{2}$. Algorithm B performs more favourably with a closer estimate to the true distribution as well as the true burnt area. However it slightly underestimates the mean due to the under-estimation of $\hat{P}_{b}$ within the burn scars. Algorithm B shows a slight over-
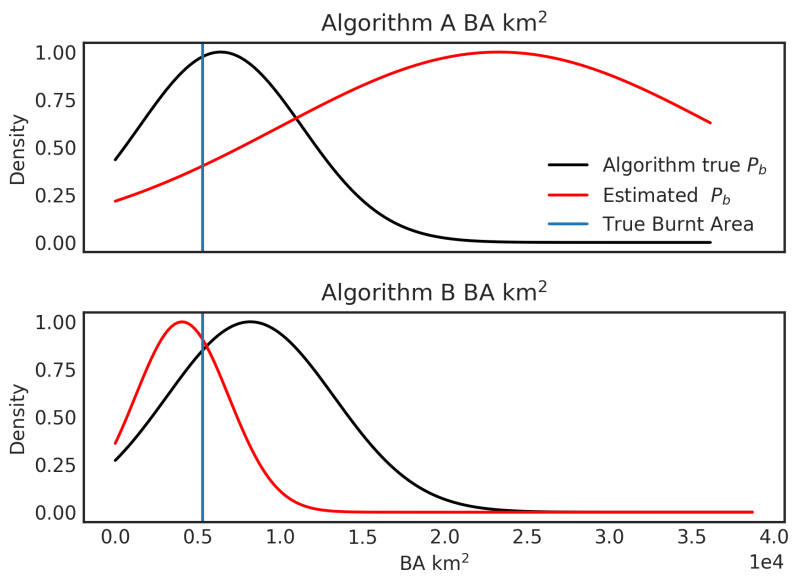

Fig. 3: Climate Model Grid (CMG) scale estimates of Burnt Area $\mathrm{km}^{2}$ for two Fire-CCI algorithms.

estimation in the standard uncertainty but is closer to the true standard uncertainty. Estimated distribution: $\mu: 4050 \mathrm{~km}^{2} \sigma$ : $2838 \mathrm{~km}^{2}$ vs the sampling-based distribution: $\mu: 8195.5 \mathrm{~km}^{2}$ $\sigma: 5077 \mathrm{~km}^{2}$.

Figure 4 shows the error in the algorithm uncertainty estimates for the three test sites. Here, the error in the uncertainty characterisation is encoded by the difference between the algorithm $P_{b}$ estimate and the true $P_{b}$ from the sampling. Generally therefore, an accurate uncertainty characterisation occurs when this error is near zero. Further, we would expect no biases in this error as a function of the noise factor $c$. Such an outcome indicates that the uncertainty method correctly identifies the magnitude of the uncertainty in the observations. A general feature of note is the overall bias in algorithm B towards an underestimate of $P_{b}$ as was seen for the boreal site in figure 2. An encouraging feature for algorithm $B$ is that the error in $P_{b}$ is not a feature of the degree of noise imposed by $c$. We can see that both the bias and distribution of errors in $P_{b}$ remain consistent across noise levels. This indicates that the algorithm provides an estimate of the uncertainty which is not sensitive to the noise sampling of the input datasets. Algorithm A shows a larger sensitivity to the noise factor $c$. In each test site, the distribution of errors in $P_{b}$ is sensitive to the level of noise $c$. This indicates that the present uncertainty characterisation needs to be refined to remove such sensitivity. Further, for algorithm A the biases in $P_{b}$ are inconsistent across the three sites unlike for algorithm B. In the boreal site, 

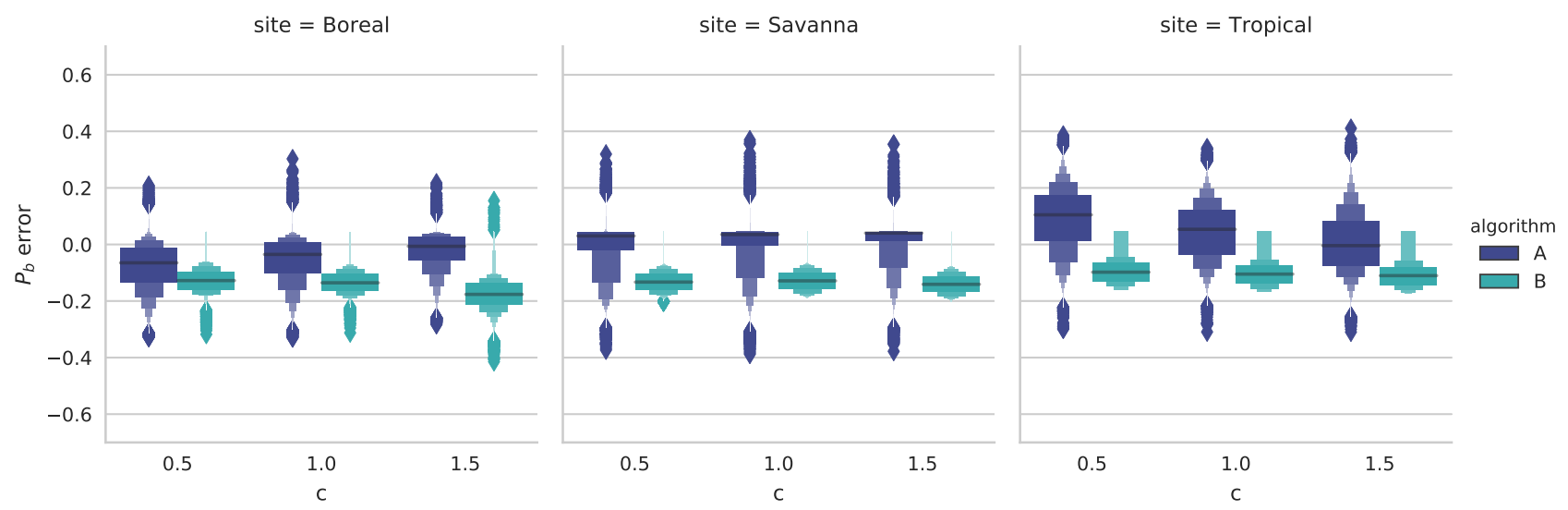

Fig. 4: Error in algorithm estimates of $P_{b}$ for the two FireCCI algorithms. $P_{b}$ error is the difference between the algorithm $P_{b}^{a}$ and the estimated true $P_{b}^{t}$.

the algorithm provided $P_{b}$ is less than the true $P_{b}$. However in the tropical and savanna sites, the algorithm $P_{b}$ is larger than the true $P_{b}$. This feature indicates that the present uncertainty method needs to be refined to be suitable for each biome.

\section{FINAL REMARKS}

The described framework represents an initial attempt at producing uncertainty quantified BA products at both the pixel and $\mathrm{CMG}$ scales. Further a methodology for validating uncertainty estimates based on sampling from the data distribution is presented. Results indicate that present uncertainty estimates need to agree more closely with the present binary estimates of products. Going forward this can be achieved by full uncertainty propagation within algorithms following metrological principles. An appropriate best practice framework for uncertainty characterisation is provided by the Guidelines for Uncertainty in Measurement (GUM) [4].

Secondly there is a need to consider uncertainty within the retrieval algorithm itself. The presented framework details the implications of the uncertainty in remote sensing observations and the effect these have on the algorithm performance, but the algorithm is here assumed to introduce no uncertainty.

\section{REFERENCES}

[1] Rainer Hollmann, Chris J Merchant, Roger Saunders, Catherine Downy, Michael Buchwitz, Anny Cazenave, Emilio Chuvieco, Pierre Defourny, Gerrit de Leeuw, René Forsberg, et al., "The esa climate change initiative: Satellite data records for essential climate variables," Bulletin of the American Meteorological Society, vol. 94, no. 10, pp. 1541-1552, 2013.

[2] AC Povey and RG Grainger, "Known and unknown unknowns: uncertainty estimation in satellite remote sens- ing," Atmospheric Measurement Techniques, vol. 8, no. 11, pp. 4699-4718, 2015.

[3] Norman D Megill and Mladen Pavicic, "Estimating bernoulli trial probability from a small sample," arXiv preprint arXiv:1105.1486, 2011.

[4] BIECIIIS IUPAC and IUPAP OIML, "Evaluation of measurement dataguide to the expression of uncertainty in measurement," 2008. 